\title{
With a national restructuring of physician training to include critical care, what is the educational value of intensive care for physician trainees?
}

\author{
Authors: Maria Paes ${ }^{\mathrm{A}}$ and Thomas Williams ${ }^{\mathrm{B}}$
}

\begin{abstract}
Introduction
Radical restructuring of physician training has been proposed from 2019 that will see core medical training replaced with the internal medicine (IM) stage 1 training programme, which will include a compulsory critical care component.

Few posts currently exist for core medical trainees (CMTs) in critical care, so there is little evidence to support how best to train physicians of this grade in the critical care environment. This study provides insight into this area, examining the educational experiences of CMTs during their intensive care unit (ICU) / highdependency unit (HDU) placements at a large teaching hospital over a 2-year period.
\end{abstract}

\section{Methods}

A mixed methods approach of questionnaires and interviews was used. All CMTs who completed an ICU post during the 2-year study period were included. Participants were CT2 at the time of the placement, thus CT2, ST3 or staff-grade specialty registrar equivalent during the study. Fifteen of 16 trainees completed questionnaires.

Inductive thematic analysis was used to analyse open question data and produce an interview guide. Semi-structured interviews explored these questionnaire themes further with eight trainees. A hybrid inductive and deductive thematic analysis method, utilising a coding framework produced from the questionnaire data, was used to structure the analysis while also coding for emergent themes. Closed questionnaire data were used to support the themes that emerged.

\section{Results and discussion}

Increased understanding of intensive treatments was a key outcome from the study and was reported to be the most valuable learning experience relevant to the CMT curriculum. CMTs were better able to understand levels of care, escalation and patient suitability and had improved awareness of negative patient outcomes following intensive intervention. Increased

Authors: A Arrowe Park Hospital; ${ }^{B}$ Wirral University Teaching Hospital NHS Foundation Trust, Liverpool, UK understanding of ICU treatments informed referral decisions on return to CMTs' general medical roles, with trainees better able to understand that reversibility and clinical need for a specific level 2 or 3 treatment were key admission criteria. Trainees already working at registrar level placed greater emphasis on targeted and appropriate referrals as a key learning outcome of their placement.

Understanding of intensive treatments was supported by situated learning and by the multidisciplinary team via a community of practice. Trainees felt that they worked more closely with both seniors and the multidisciplinary team (MDT), easily integrating into the community due to its small size. High consultant presence led to an apprenticeship style of teaching, with juniors learning alongside their consultants.

The value of learning in a senior-led environment was a further study outcome. This was reported to be the primary difference in trainees' learning experiences when asked to compare their ICU post with their learning on the general medical wards. Trainees were able to learn alongside an expert, allowing rapid development beyond baseline capabilities. Similarities to an anaesthetic approach of one-to-one teaching, grounded in a cognitive apprenticeship approach, were noted. The improved teacher-student relationship, compared with general medical experiences, facilitated teaching better aligned to learners' needs and opportunity for feedback. High senior presence was, however, associated with negative features of decreased autonomy and circumvention of juniors' opinions.

While structured handover, procedures (eg central line insertion), bedside teaching and ward work on ICU/HDU were felt to be valuable learning experiences, the key learning opportunities identified for CMTs were medical emergency team (MET) calls and taking referrals. Trainees gained experience of managing high-acuity patients in these settings. Confidence, communication and prioritisation skills in particular were felt to develop as a result. MET calls allowed trainees the opportunity to learn a systematic approach to assessing the acutely unwell patient, observe a range of medical registrar peers and lead calls. Immediate, targeted and constructive feedback was provided through discussion of each MET call and referral with an ICU consultant. Trainees then had the opportunity to apply and test learning from feedback rapidly due to the volume of MET calls and referrals received daily, with learning enhanced by this experiential learning cycle. Supported learning was valuable, but autonomy with referrals and MET calls allowed testing and reflection. 
This study showed that ICU experience supported CMTs in their transition to their medical registrar roles. Key areas of relevant development included improved complex decision making, for example regarding end of life and escalation of care, confidence and experience with managing high-acuity patients, improved referrals to ICU and experience with MET calls.

\section{Conclusions}

Critical care placements were perceived to be beneficial to core medical training. The key advantages identified were an increased understanding of intensive treatment, learning in a senior-led environment and experience of working with high-acuity patients. All three were felt to provide skills valuable in supporting transition to specialty medical registrar training.

This study provides insight into provision of training for internal medicine trainees in critical care under the proposed restructuring of medical specialty training. 